\title{
LE SCELTE DANTESCHE DI ALEJO CARPENTIER
}

\author{
María Teresa NaVArRo Salazar \\ UNED. Madrid \\ mnavarro@flog.uned.es
}

Miro al cielo como nauchero desnortado que pide su rumbo a las costelaciones.

A. Carpentier, La consagración de la primavera

\section{RESUMEN}

Dante Alighieri sigue siendo hoy un autor vital, modelo en vigor para la literatura de recepción. Reflejo de su modernidad son los ejemplos de intertextualidad activos en el panorama literario universal, en el que la Divina Comedia y, en especial el Infierno, forman parte de un acervo cultural común, fuente de inspiración para muchos autores contemporáneos.

PALABRAS ClAVE: intertextualidad, Dante, Infierno, Carpentier.

\section{RIASSUNTO}

Dante Alighieri rimane sempre un autore moderno, modello per la letteratura di ricezione. La sua vitalità si percepisce nei tanti esempi di intertestualità, rintracciabili nel panorama letterario universale. La Commedia e, in modo speciale l'Inferno, fanno parte di un insieme culturale comune che ispira molti autori contemporanei.

PAROLE CHIAVI: intertestualità, Dante, Inferno, Carpentier. 
Dante Alighieri è oggi, sicuramente, uno fra gli autori più visibili all'interno di quel complesso insieme, noto come letteratura di ricezione ${ }^{1}$ e, lungo i secoli, è sempre rimasto un ricercato referente. Traslasciando ora il resto della sua produzione letteraria, prenderemo in esame qualche passo della Commedia, origine di rilevanti esempi di intertestualità ${ }^{2}$, espressi in forme, livelli, e lingue differenti. Per ciò che riguarda l'area ispana ${ }^{3}$, proprio il primo verso dell'Inferno, riscritto da Rubén Darío:

En medio del camino de la vida

dijo Dante, y su verso se convierte:

en medio del camino de la muerte.

è servito come spunto a José Bergamín per una accorta riflessione «sobre Dante, y su Infierno como ilusión de mito» ${ }^{4}$, e in area ispana rimarremo per illustrare la vitalità del poeta fiorentino, considerando le scelte dantesche fatte da Alejo Carpentier.

La produzione letteraria dello scrittore cubano ${ }^{5}$ è stata studiata da parte della critica, valutando le molteplici fonti d'ispirazione di cui lui si è avvalso nella costruzione di un mondo creativo, fortemente connesso alla descrizione della realtà latinoamericana. Questo disegno narrativo sbocca in uno stile particolare, imperniato sulla teoria che lui stesso ha definito: lo real maravilloso ${ }^{6}$, «polisémico fenómeno» che, a seguire Padura, non potrà essere interpretato senza prendere in analisi le tappe ${ }^{7}$ dove si coglie il progressivo evolversi di tutti i componenti. Di recente Dario Villanueva ${ }^{8}$ ha inserito un nuovo elemento nella scacchiera della teoria che riguarda lo real maravilloso.

Sono note la personalità e la cultura universale di Carpentier, nel quale è possibile scorgere una linea di azione che punta verso la ricerca della propria identità, fissata, spesso, nella ricchezza linguistica pregnante di un estremo barocchismo ${ }^{9}$ che accompagna le

${ }^{1}$ Per il concetto di ricezione, si veda: José Enrique Martínez Fernández La intertextualidad literaria. (Base teórica y práctica textual), Madrid, Cátedra, 2001, pp. 66-69.

2 Si veda: Peter Kuon, «Lo mio maestro e'l mio autore» Die produktive Rezeption der «Divina Commedia» in der Erzählliteratur der Moderne, Frankfurt am Main, Vittorio Klostermann, 1993.

${ }^{3}$ Si veda in propósito: Joaquín Arce, «Dante nel Novecento spagnolo», in Il Veltro, 1968, XII, n. 6 e Ídem «Studi ispano-danteschi: rassegna bibliografico-critica (1965-1977)», in Studi di letteratura italiana in onore di Fausto Montanari, Genova, Il Melangolo, 1980.

4 José Bergamín: «Dante», en Fronteras infernales de la poesía, p. 33.

5 (La Habana, 1904-París, 1980): «Premio Cervantes de las Letras Españolas» (1977) e Premio Medici (1979). Alejo Carpentier: Obras completas, 9 vols. México, Siglo XXI, 1983-86. Si veda anche Klaus Müller Bergh, Alejo Carpentier: estudio biográfico-crítico, Nueva York, Las Américas, 1972 e Araceli García Carranza, Bibliografía de Alejo Carpentier, La Habana, Letras cubanas, 1984.

6 Alejo Carpentier. Prefazione a El reino de este mundo, México, E.D.I.A.P.S.A.,1949, (si cita dall'edizione El reino de este mundo, Barcelona, Edhasa, 1978, pp. 52-57).

7 Padura Fuentes, Leonardo: Un camino de medio siglo : Alejo Carpentier y la narrativa de lo real maravilloso, pp. 202-203.

${ }^{8} \mathrm{Si}$ tratta del rapporto da stabilire fra questa e i viaggi e le scoperte del francescano Francisco Menéndez. Darío Villanueva La referencia compostelana en la teoría de lo real maravilloso de Alejo Carpentier pp. 144-145.

9 Nella definizione di Eugenio D’Ors: «... una suerte de pulsión creadora, que vuelve cíclicamente a través de toda la historia en las manifestaciones del arte, tanto literarias como plásticas...»; «...un arte en movimiento, un arte de pulsión, un arte que va de un centro hacia fuera y va rompiendo, en cierto modo sus propios márgenes»; «¿Y por qué es América Latina la tierra de eleción del barroco? Porque toda simbiosis, todo mestizaje, engendra un barroquismo.» Alejo Carpentier «Lo barroco y lo real maravilloso». Conferencia dictada en el Ateneo 
descrizioni del rigoglioso paesaggio latinoamericano dove vengono inseriti i personaggi dei romanzi e i racconti.

Figlio di un francese e di una russa, dal padre e dagli studi liceali realizzati in Francia riceve in eredità una formazione razionalistica improntata sul pensiero di Descartes, autore che ha sempre ammirato e, a riprova, il titolo di un suo romanzo è proprio El recurso del método. La madre, invece, lo mette a contatto con il mondo culturale russo, del quale Carpentier assorbisce forme precise che hanno a che fare con la letteratura e la musica. Il titolo di un altro suo romanzo: La consagración de la primavera ci dà compiuta conferma dell'assunzione di elementi attinenti a questi due campi. Ma non solo, la profondità delle sue conoscenze musicali e la formazione musicologica si palesa in Los pasos perdidos, romanzo che serve all'autore per avviarsi alla ricerca di certi singoli strumenti musicali primitivi, sostenuto dalla speranza di poter trovare e, quindi provare, la teoria della nascita dell'espressione ritmica primigenia, che andrebbe ritrovata «a base de mi ingeniosa teoría del mimetismo-mágico-rítmico» ${ }^{10}$. Alla ricerca di questi suoni s'inoltra nella «gran Selva del Sur».

Partendo da El reino de este mundo ${ }^{11}$ (1949), nella lunga produzione che si profila fino alla redazione di El arpa y la sombra (1979), Carpentier si pone l'obbiettivo di far conoscere la realtà della cultura latinoamericana. Insiste, poi, nel mettere in evidenza l'inalterata continuità, visibile nel nuovo mondo, di «una conciencia de muy viejas tradiciones, un recuerdo vivo de ciertos mitos que era en suma, presencia de una cultura más honrada y válida, probablemente, que la que se nos había quedado allá». E rincara la dose con l'affermazione: «Para un pueblo era más interesante conservar la memoria de la Canción de Rolando que tener agua caliente a domicilio» ${ }^{12}$.

In seguito alla sua morte, Rafael Conte scriveva che il nostro autore «supo combinar en el terreno expresivo tres herencias dispares —el castellano clásico, el surrealismo y el barroco americano- fundiéndolas como nadie en un resultado perfectamente unitario y espléndido, su inigualable prosa narrativa» ${ }^{13}$. A queste eredità ne aggiungeva Vázquez Rial ${ }^{14}$ diverse altre: Carpentier critico dell' 'Illustrazione ed il Romanticismo, Carpentier organizzatore culturale nella pratica rivoluzionaria, Carpentier musicista, Carpentier erudito spagnolo, Carpentier francese e cartesiano ribelle.

Orbene, tra i ricchi e diversi influssi or ora accennati, sembra che la critica abbia stentato a collocare, allo stesso livello di tutte le altre, l'eredità culturale italiana che permea tutta la sua produzione letteraria. Diffatti, come si cercherà di esporre, la forte e voluta presenza del mondo e della cultura italiana è rintracciabile in situazioni e livelli diversi.

de Caracas, el 22 de mayo de 1975. Ora in La novela latinoamericana en vísperas de un nuevo siglo y otros ensayos, p. 113, 117 e 126.

${ }_{10}$ Alejo Carpentier: Los pasos perdidos, México, Edición y Distribución Iberoamericana de Publicaciones, Autores Iberoamericanos Contemporáneos, 1953. Si cita da: Los pasos perdidos, Barcelona, Barral Editores, 1972, p. 24.

${ }_{11}$ Per la produzione anteriore si veda Frank Janney Alejo Carpentier and his early works, London, Tamesis Books Limited, 1981.

${ }^{12}$ Los pasos perdidos, o. cit., p. 123.

${ }^{13}$ El País 11-V-1980. Citato da Horacio Vázquez Rial, Camp de l'arpa. Revista de literatura, 88, Junio 1981, p. 5. Si tratta di un monografico su Alejo Carpentier.

${ }^{14}$ Horacio Vázquez Rial, Ibidem, p. 6. 
Il rapporto linguistico, letterario e culturale che lega Carpentier alla civiltà italiana è antico e provato, ben reperibile nelle sue opere: personaggi e istituzioni di varie epoche e attività compaiono, con notevole dimestichezza, in romanzi, saggi e racconti ${ }^{15}$. Questo rapporto, che esemplificheremo partendo dal romanzo Concierto barroco (1974), si sviluppa in una tonalità ascendente: movendo dall'immissione del lessico specifico musicale italiano che costella l'opera, si allarga in continui riferimenti a diversi passi dell'Inferno in El arpa y la sombra (1979), ultimo lavoro consegnato alla stampa pochi mesi prima della morte, a cui è stato assegnato il valore simbolico di chiave interpretativa del suo universo narrativo ${ }^{16}$. Il processo di ricezione acquisisce una consistenza più significativa in altre due opere: il racconto El derecho de asilo (1972), e il romanzo Los pasos perdidos (1953); qui l'intertestualità dantesca non è più fondata su riferimenti isolati, ma riguarda precise e compiute strutture della Commedia. Nel racconto Carpentier rivisita il noto episodio di Paolo e Francesca, ma la scena dei due famosi amanti viene ricreata, non soltanto in contesti temporali e geofisici molto diversi, ma anche nel segno di un bizzarro umorismo. Nel romanzo, invece, a mio parere, credo si possa intuire il riferimento a un'altra struttura dell'Inferno e, più precisamente, a quell'ampio cono a forma di imbuto che in sé contiene il male e il dolore dell'intiera umanità.

\section{CONCIERTO BARROCO}

Collegato alla sua passione di studioso e teorico ${ }^{17}$ della musica nasce il breve romanzo Concierto barroco ${ }^{18}$. Il protagonista, un nobile messicano, travestito da Moctezuma, ispira a Vivaldi l'omonima opera ${ }^{19}$. Nell'ambito di un sincretismo culturale e temporale, certamente novissimo, vediamo seduti insieme intorno a una tavola i musicisti Vivaldi (Venezia, 1680? - Vienna, 1714), Domenico Scarlatti (Palermo, 1660 - Napoli, 1725), e Händel (Halle, 1685 - Londra, 1759) che condividono la loro prima colazione con il nobile messicano Moctezuma, affiancato dal suo servo Filomeno. Questa eccezionale colazione si svolge niente di meno al cimitero di Venezia, davanti alla tomba di Stravinsky. Ragionando insieme al tempo che addentano «una tajada de morro de jabalí escabechado en vinagre, orégano y pimentón» ${ }^{20}$, Händel esprime il suo parere a proposito

15 Sono ricorrenti, ad esempio, i nomi di Dante, Petrarca, Campanella, Giordano Bruno, D’Annunzio, Michelangelo, Paolo Ucello, Cellini, Bramante, Vignola, Brunelleschi, Canova, Piranesi, Pergolesi, Cimarosa, Monteverdi, Vivaldi, Cesare Cantù, ecc.

16 Dámaso Santos: «Alejo Carpentier», en Sábado Literario, Suplemento semanal del diario Pueblo, 26 de abril, 1980.

${ }^{17} \mathrm{Su}$ Carpentier musicista e teorico della musica si veda Alejo Carpentier. Ese músico que llevo dentro y La música en Cuba (1987 [1946]), Madrid, Siglo XXI. Si è occupato anche di opera italiana: Su Mascagni e l'opera verista : «Una mala aventura de Lazaro y del autor de Cavallería rusticana» en Carteles, 20-I- 1929. Ora in Alejo Carpentier Bajo el signo de la Cibeles, pp. 33-37.

18 Alejo Carpentier: Concierto barroco, México, Siglo XXI Editores, 1974. Si cita da Concierto barroco, Madrid, Siglo XXI Editores, 1974.

${ }^{19}$ L'interesse di Carpentier per questo tema era nato molto prima, quando nel 1936 viene a sapere da Francesco Malipiero che Vivaldi aveva scritto un'opera sulla conquista dell' America. «Habla Alejo Carpentier», in Recopilación de textos sobre Alejo Carpentier, p. 40.

${ }^{20}$ Concierto barroco, o. cit., p. 52. 
dei temi musicali di Stravinsky che ritiene non originali, occasione che il musicologo Carpentier sfrutta per disegnare una lunga linea d'interpretazione musicale. Suggerisce, infatti, una linea che inizia con la giovane «Cattarina del Cornetto», allieva di Vivaldi all'Ospedale della pietà, che poi si congiungerà — facendo da tramite il negro Filomeno virtuoso suonatore di tromba-, al «cobre impar de Louis Amstrong», riproponendo un duraturo leitmotiv che andrebbe interpretato quale invito a una migliore intesa fra il Vecchio e il Nuovo mondo ${ }^{21}$. In questa estrosa opera che è Concierto barroco sono molteplici i richiami (diamo soltanto qualche esempio) alla cultura $\operatorname{artistica~}^{22}$, popolare ${ }^{23}$ e musicale $^{24}$ dell'Italia rinascimentale e barocca, a noti luoghi ${ }^{25}$ della città di Venezia e anche a «...el calor de la segrete cose cantada por el Dante» ${ }^{26}$.

Nell'assunzione di lessico specifico, la riproduzione di ambienti, o la ricreazione di situazioni concernenti la vita e le tradizioni italiane si può individuare un approccio deciso di Carpentier verso l'arte e la letteratura italiana. Il vincolo s'infittisce quando, poi, in El arpa y la sombra, in El derecho de asilo e ancora in Los pasos perdidos l'autore si rifà a precisi modelli di intertestualità dantesca.

\section{EL ARPA Y LA SOMBRA}

In modo non dissimile a quanto aveva fatto in Concierto barrocco la cultura italiana ha una vastissima presenza in El arpa y la sombra. Il titolo riprende una citazione dalla Leggenda aurea del domenicano genovese Jacopo da Varazze ${ }^{27}$ : «En el arpa, cuando resuena, hay tres cosas: el arte, la mano, la cuerda. En el hombre: el cuerpo, el alma, la sombra», inserita da Carpentier quale incipit della narrazione, e poi, lungo tutto il romanzo, vediamo comparire chiese, artisti, viaggiatori, santi, e personaggi diversi ${ }^{28}$, appartenenti

21 «Según el Preste Antonio, todo lo de allá es fábula.» - «De fábulas se alimenta la Gran Historia, no te olvides de ello. Fábula parece lo nuestro a las gentes de acá porque han perdido el secreto de lo fabuloso. Llaman fabuloso cuanto es remoto, irracional, situado en el ayer — marcó el indiano con pausa—: No entienden que lo fabuloso está en el futuro. Todo futuro es fabuloso». Ibidem, p. 77.

22 Allusione alla pittrice Rosalba Carriera (1675-17579 «... aparecían tres figuras debidas al pincel de $R o$ salba pittora, artista veneciana.... » Ibidem, pp. 11-12.

${ }^{23}$ «... un juego de naipes, de estilo desconocido aquí, llamado minchiate, inventado por el pintor Miguel Ángel, según decían para enseñar aritmética a los niños... » Ibidem, p. 15; «... que allí los calificativos de coglione e hijo de la grandísima puta venían a quedar en lo más liviano del repertorio». Ibidem, p. 39.

${ }^{24}$ Un lungo elenco di strumenti musicali, Ibidem, pp. 42-43-44. «El poeta Alvise Giusti, autor de este "drama para música"...» Ibidem, p. 68. Alvise Giusti fue el libretista de la ópera Motezuma que con música de Vivaldi se estrenó en Venecia en 1733. «Si tanto le gustan las fábulas, ponga música al Orlando Furioso». Ibidem, p.70

25 «... el Amo, vestido de Monctezuma, entró en la Botteghe di Caffé (sic) de Victoria Arduino... ». Ibidem, p. 35. Si riallaccia al futuro facendo riferimento al Palazzo Vendramin-Calergi dove è vissuto Richard Wagner e alla sua morte: «[el ataúd] es de un músico alemán que murió ayer de apoplejía», Ibidem, p. 56.

26 Ibidem, p. 13.

27 Jacopo Da Varazze: Legenda aurea, vulgo Historia Lombardica dicta (1845). Osnabrück, Otto Zeller, Verlag, 1969.

${ }^{28}$ El arpa y la sombra, Madrid, Siglo Veintiuno de España editores, S.A, 1979. Chiese e monumenti, chiesa del Gesú, Santa Maria Maggiore, Santa Maria sopra Minerva, Ibidem, p. 15; Santa Maria di Loreto nella Marca di Ancona, Ibidem, p. 142; la Cappella Sistina, Ibidem, p.192; la Colonnata di Bernini, Ibidem, p. 191; Cultura italiana: Muzio Clementi, Ibidem, p. 22, Pergolesi, Ibidem, p. 34 e 40, Palestrina, Ibidem, p. 40; Michelangelo, Ibidem, p. 15; Bronzino, Ibidem, p. 20 e 222; Benvenuto Cellini, Ibidem, p. 18; Brunelleschi, Ibidem, 
all'universo culturale italiano. Ma, insieme a luoghi, fatti, strumenti e personaggi che rappresentano l'Italia nella sua dovizia culturale, diventata ormai patrimonio universale, di cui Carpentier si serve in citazioni sparse lungo tutta l'opera, alla ricezione dantesca viene concesso uno spazio altamente significativo, poiché la Commedia è presente in passi tratti da: Inferno IV, V, e XXXIII e Purgatorio XXX .

Lo sfondo narrativo di El arpa y la sombra si svolge intorno all'ucronica beatificazione dell'ammiraglio Cristoforo Colombo ${ }^{29}$, che non è mai arrivata in porto, malgrado la potenza dei suoi postulatori: i pontefici Pio IX e Leone XIII, appoggiati anche da 850 vescovi, perché la Sacra Congregazione per i Riti non ha voluto mai accettare tale postulazione. Rifacendosi a Aristotele, Carpentier ritiene che mestiere del romanziere non è «el contar las cosas como sucedieron, sino como debieron o pudieron haber sucedido» e in seguito chiarisce quale è i suo concetto di invenzione: «Este pequeño libro sólo debe verse como una variación (en el sentido musical del término) sobre un gran tema que sigue siendo, por lo demás misteriosísimo tema... $\rangle^{30}$.

È cosí che Cristoforo Colombo alla ricerca di «la tierra del vino» diventa un nuovo Giasone che insegue il Vello d'oro oppure un nuovo Ulisse alla ricerca delle verità nascoste aldilà delle colonne di Ercole. La scelta dell'uomo che legherà per la prima volta vecchio e nuovo continente non è, quindi, casuale e gli permetterà di «...ironizzare sul Real maravilloso, che viene trasfigurato [...] in menzogna..... $\rangle^{31}$. Ormai, si sa, il processo di beatificazione non è mai giunto a termine. In El arpa y la sombra vediamo Colombo, soprannominato l'Invisibile, esser presente nella sala dove si tiene il processo, che intreccia i propri commenti alle parole dette dal Postulatore, Giuseppe Baldi, genovese pure lui, dal Promotor fidei, dal Protonotaro, e dal Presidente della commissione, ai quali dedica alternativamente brutte invettive, oppure smisurate lodi a seconda dell'assenso o rigetto espressi sulla sua causa. Il verdetto emesso dalla Commissione ha, come prevedibile, un esito negativo. In fondo Colombo altro non è che un marinaio genovese, pure ammiraglio, ma certamente negriero e truffatore, persona non affidabile che dice di se stesso: «llegada la hora de la verdad me pongo la máscara de quién quise ser y no fui $»^{32}$, noncurante di tradire la fiducia che in lui ha riposto la regina Cattolica. Non merita, quindi, il titolo di beato, ma piuttosto un luogo fra i traditori.

Finito il fasullo processo di beatificazione, l'Ammiraglio della Regina di Spagna, deluso e accasciato, esce sulla piazza di San Pietro e incontra un altro invisibile, conterraneo e quasi coetaneo, che gli parla nel loro comune dialetto: il genovese. È Andrea Doria, il grande ammiraglio, che difese la città di Roma dalla cupidigia del re francese

p.211; Fra Angelico, Ibidem, p. 223 i goliardi e le fiere d'Italia, Ibidem, p. 87; la mappa di Toscanelli Ibidem, p. 110, Oderico de Pordenone, Ibidem, p. 119 e 137; Il Santo di Assisi e Madonna povertà, Ibidem, p. 139, San Antonio de Padua, Ibidem, p.195; una Sacra rappresentazione, Ibidem, p.178; Marco Polo, Ibidem, p. 137; Americo Vespucci, Ibidem, p. 223 et passim. citazioni: . «No tenemos un solo santo marinero. Por más que he buscado en La Leyenda Áurea [...] no hallo uno solo», Ibidem, p. 193; «¡Absalón! ¡Ugolino! ¡Judas Iscariote! ¡Escoria del mundo! » Ibidem, p. 208.

${ }^{29}$ Racconta l'autore che lo spunto risale al 1937 quando realizza l'adattamento per la radio di Il libro de Cristobal Colón de Claudel. Alejo Carpentier La novela latinoamericana en vísperas de un nuevo siglo y otros ensayos, o. cit. quarta di copertina.

${ }^{30}$ Ibidem, quarta di copertina.

${ }^{31}$ Chiara Zamborlin La narrativa di Alejo Carpentier: il mito del mondo migliore, p. 143.

${ }^{32}$ El arpa y la sombra, o. cit. p.186. 
Francesco I, e parteggiò per la Spagna passando al servizio di Carlo V. Il dialogo stabilito fra di loro diventa modello di invettive e accuse vicendevoli; ricorda il Doria, ritenuto «el mayor azote de los bajeles del turco $»^{33}$, che lui è ammiraglio non di viaggi, ma di battaglie e si rifà alla spaventevole maledizione dedita da Dante ai genovesi ${ }^{34}$ nel Canto XXXIII dell' Inferno: «¡Ah, genoveses! Hombres ajenos a toda buena costumbre y repletos de vicios... ipor qué no sois arrojados de la tierra? ».

Ma Andrea Doria, interlocutore di Colombo, non è l'unico personaggio di questa nobile famiglia genovese individuabile in El arpa y la sombra, perché, accanto a lui, Carpentier vuole anche ricordare il crudele assassino Branca Doria ${ }^{35}$, che espia la pena inflitta nel nono cerchio dell'Inferno, nel girone dei frodolenti in chi si fida. Lì, nella terza zona, Tolomea, Dante situa Branca Doria, uccisore a tradimento del suocero, Michele Zanche, che aveva in mano la Signoria di Logodoro ${ }^{36}$. Per ambizione politica, volendo il Doria diventare signore di Logodoro, invitò a cena suo suocero e ammazzò lui e «tutta sua compagnia». Questo tradimento viene considerato talmente grave dall' Alighieri che gli assegna un orrendo contrappasso: separa il suo corpo dalla sua anima. Infatti, quando nel aprile del 1300, Dante scende nell'Inferno il Doria vive ancora e non dovrebbe essere annoverato fra i morti, ma per Dante l'anima del Doria è già giunta nell'inferno e, nonostante, il suo corpo sembri essere ancora vivo, in realtà ha soltanto una parvenza di vita, poiché è possieduto dal demonio e, quindi, morto per la salvezza.

Largamente había meditado el joven ante la casa de Branca Doria, aquel muy magnífico asesino, de estirpe genovesa, a quien Dante halló en el noveno círculo infernal, padeciendo su castigo en alma mientras su cuerpo, movido por un demonio, se mostraba aún viviente sobre la tierra, pp. 31-32.
E perché tu piú volontier mi rade le'nvetriate lacrime del volto, sappie che tosto che l'anima trade come fec'io il corpo suo l'è tolto da un demonio, che poscia il governa mentre che ' 1 tempo suo tutto sia vòlto.

Inferno, XXXIII, vv. 127-132.

Il nono cerchio dell'Inferno è stato anche rivisitato dal siciliano Giuseppe Bonaviri ${ }^{37}$ nel suo romanzo Silvinia ${ }^{38}$. Ma, il contrasto fra l'autore cubano e lo scrittore italiano nel modo di servirsi e interpretare il testo dantesco è talmente discordante, che mi è sembrato

33 Ibidem, p. 225.

${ }^{34}$ Ibidem, p. 225. «Ahí genovesi, uomini diversi/D’ogne costume e pien d'ogni magagna, / Perché non siete voi del mondo spersi? »Inferno, XXXIII, vv. 151-153.

35 «Tu ' 1 dei saper, se tu vien pur mo giuso:/elli è ser Branca Doria, e son più anni/poscia passati ch'el fu sì racchiuso»./ «Io credo», diss'io lui, «che tu m'inganni;/ché Branca Doria non morì unquanche,/e mangia e bee e dorme e veste panni»./ «Nel fosso sù», diss'el, «de' Malebranche,/là dove bolle la tenace pece,/ non era ancor giunto Michel Zanche,/ che questi lasciò il diavolo in sua vece/nel corpo suo, ed un suo prossimano/he ' 1 tradimento insieme con lui fece». Inferno, XXXIII, vv. 136-147.

36 Il fatto avvenne verso il 1275 secondo certi storici, verso il 1290 credono altri.

${ }^{37}$ L'episodio del maelstrom qui riportato è tratto, con diverse modificazioni, da: María Teresa Navarro, «Scritture sul mare: Bonaviri e Silvinia», pp. 352-357.

${ }^{38}$ Giuseppe Bonaviri, Silvinia, Milano, Mondadori, 1997. Prima fra le sue opere tradotte in spagnolo, è stata presentata all' UNED nell'ottobre del 1998. 
lecito fare qui il paragone. Quando la piccola Silvinia sparisce, il padre, alla ricerca della figlia, s'imbarca alla volta di New York sulla nave Madonna e, durante la traversata dell'Atlantico i passeggeri s'imbattono nella spaventosa corrente del maelstrom, fenomeno certo non comune nelle acque dell'Atlantico. Nel capitolo XXVIII di Silvinia ${ }^{39}$ Bonaviri descrive il maesltrom e, partendo dal noto racconto di Poe, A Descent into the Maelströ $m^{40}$, risale all'Inferno dantesco, sia nell'etica della punizione, sia nella scelta di certi modelli di contrappasso.

Difatti, in rotta verso New York, il comandante informa i passeggeri che sono sul punto di arrivare al mare dei Sargassi nell'Atlantico Nord. Lì l'incontro della corrente del Golfo, cioè la Gulf Stream, con quella più fredda che viene da Nord, la corrente del Labrador, provoca i turbini vorticosi, che Bonaviri soprannomina maelstrom. Ma in Silvinia l'orrendo baratro si crea partendo dalla commistione delle due correnti fisiche accennate, e un'altra, simbolica, quella dei Wrongdoers' Stream: cioè, la Corrente dei malfattori. «In seconda giornata, quando arrivò l'aurora, pervennero nel punto centrale della corrente detta Wrongdoers' Stream [...] Era un immenso fiume che solcava ${ }^{41}$ il mare, dal quale si differenziava per un colore blu intenso». La presenza di questi tre corsi d'acqua sembra richiamare altre tre correnti infernali: Acheronte, Flegetonte e Cocito. Ma non soltanto, questo travolgente e impetuoso vortice che, attraverso fiumi diversi, riceve i corpi in disfacimento di tutti i malfattori del mondo, diventerà per essi luogo dove scontare il loro castigo.

In verità, come la stessa espressione Wrongdoers' Stream dice, era una fiumana di malviventi che, deceduti in pochi anni, scendevano verso l'oceano trasportati dal Nilo, dal Po, dal Volga, dal Danubio, dalla Senna, dal Tamigi, o vi arrivavano attraverso le acque del Mississippi, dell'Orinoco, dal Rio delle Amazzoni. Altri provenivano dal Fiume Giallo, dal Tevere, e dai gran torrenti che si formano dalla neve del Fujiyama ${ }^{42}$.

Sicuramente, è nelle pene che vediamo infliggere a questi malviventi, sballottati senza misericordia dalle inferocite acque dell'Atlantico, dove la ricezione dantesca diventa più significativa. Allo stesso modo dei dannati della Commedia, i criminali di Bonaviri sono divisi in schiere separate secondo le colpe di cui si sono fatti responsabili in vita: «Si trattava di politici corrotti, di trafficanti di droga, di omicidi, di mafiosi, di rapitori di bimbi, di gente usa a adorare il denaro, di industriali, e di piccole donne, e mogli illusorie, e omuncoli vissuti nel guscio della loro esistenza» ${ }^{43}$.

I malfattori di Bonaviri hanno tradito le persone, gli elettori, i partiti e, persino, la loro patria. È stato detto che, per Dante, «da ogni pagina di questi due canti XXXII e XXXIII deve scaturire risoluta la condanna, non già di questa o quella parte politica, ma dei metodi e delle forme di tutta la politica dell'età comunale, vista e giudicata nel

\footnotetext{
${ }^{39}$ Ibidem, pp. 144-152.

40 Edgard Allan Poe, Poe. Poetry and Tales, New York, The Library of America, 1984, pp. 432-448. Pubblicato per la prima volta nel Graham's Magazine nel maggio del 1841.

${ }^{41}$ Silvinia, O. cit., p. 146. Tranne la forma Wrongdoers' Stream, che compare sempre in corsivo nel romanzo, il corsivo all'interno delle citazioni è nostro.

42 Ibidem, p. 148.

43 Ibidem, p. 148.
} 
suo complesso» ${ }^{44}$. Condanna questa che Bonaviri pare voglia allargare alla vita politica dei nostri tempi. I violenti e traditori ricevono ora il contrappasso, la pena di essere immersi nell'acqua, rivolti in su, con le palpebre righe livide socchiuse alla luce del bene.

Era un immenso fiume che solcava il mare, dal quale si differenziava per un colore blu intenso. In quella corrente, misti alle alghe del Mar dei Sargassi, galleggiavano dei corpi, milioni di corpi dall'addome gonfio e dalla faccia verde. Nei giovani la bellezza era in disfacimento, nei vecchi canuti cominciava la putrefazione. Le palpebre, righe livide, erano socchiuse, le pupille erano atrofiche, piccolissime, perché non avevano mai visto la luce di $\mathrm{Dio}^{45}$.
Noi passamo oltre, là 've la gelata ruvidamente un'altra gente fascia, non volta in giù, ma tutta riversata. Lo pianto stesso lí pianger non lascia, e il duol che trova in su li occhi rintoppo, si volge in entro a far crescere l' ambascia; ché le lagrime prime fanno groppo, e sí come visiere di cristallo, riempion sotto 'l ciglio tutto il coppo. Inferno, XXXIII, vv. 91- 99.

Si palesa che sia Bonaviri che Carpentier hanno in mente il modello testuale dantesco che si riferisce ai traditori del nono cerchio, ma sorprende quanto la loro ricezione possa essere divergente. In Bonaviri si coglie la fedeltà allo spirito dantesco, e nel contrappasso che saranno costretti a subire i malfattori, e nel valore di exemplum assegnato a questo episodio, nel quale, sulle orme di Dante e a distanza di secoli, ripropone una forte critica verso la corruzione politica odierna e quelli che si servono del potere. Simbolicamente, Bonaviri condanna tutti i malviventi del mondo, facendoli inabissare nelle torbide acque dell'oceano Atlantico, poiché per lui, uomo attento ai segni d'identità che fissano la vita di ogni individuo, la più grave delle punizioni risiede nella perdita delle proprie origini e lí «sul mare vengono a mancare i riferimenti, le radici» ${ }^{46}$.

Carpentier, invece, reinterpreta Dante a modo suo. Il testo perde lo spessore ideologico e diventa una citazione, quasi a sfoggio culturale. Nessun riferimento al raccapricciante contrappasso e nemmeno un'allusione al contesto storico e politico che regola la vicenda del personaggio; in questo modo l'accenno a Branca Doria, rimane un ricordo letterario isolato, lontano da qualsiasi implicazione ideologica, quasi un semplice referente spaziale. Stupisce, certamente, questo modo di riproporre l'episodio dantesco da parte di un autore impegnato, da un punto di vista storico e politico, quale è stato lo scrittore cubano. Per capire questo atteggiamento tornerebbero forse utili le sue stesse parole, concernenti la Commedia: «...o me interesaba por saber si aquel que se había extraviado en una selva oscura al alcanzar el medio tránsito de su vida había podido salir del atolladero, a pesar de las tres alimañas que lo molestaban. Vivía, una vez más fuera de la

${ }^{44}$ La divina commedia, Inferno, a cura di Natalino Sapegno, Firenze, La Nuova Italia Editrice, $1967^{20}$, p. 363 nota 80 .

45 Silvinia, o. cit., p.147.

46 Roberto Alonge, Luigi Pirandello, Roma. Laterza, 1997, pp. 112-113. 
Época ${ }^{47} »$, in cui, in coincidenza con quanto appare in El derecho de asilo, fornisce un'interpretazione in chiave umoristica ${ }^{48}$.

La Commedia è ancora presente all'inizio della parte III del romanzo El arpa y la sombra che riprende nell'incipit un verso del primo Cerchio dell'Inferno, il Limbo, dove i grandi spiriti dell'antichità desiderano invano di vedere Dio: [...] «Tu non dimandi/che spiriti son queste [sic] che tu vedi? ${ }^{49}$. In seguito, quando l'avvocato del diavolo accenna agli amori illegitimi di Colombo con una certa Beatrice, l'ammiraglio si commuove e fa suoi i versi di Dante che, vedendo apparir Beatrice ${ }^{50}$ in riva al fiume Lete, sente rivivere il suo cuore gelato «... el hielo que se había endurecido en torno a mi corazón se hizo suspiros y lágrimas, brotando de mis entrañas, apresurado, por la boca y por los ojos... ». Con il pensiero ancora rivolto alla sua Beatrice, Colombo, si ferma davanti ad una tela con un San Sebastiano trapassato da crudeli saette e considera che anche lui stesso è stato violentemente ferito da altre saette, quelle che trafiggono gli eletti, quelle che spingono gli amanti nel «huracán infernal $»^{51}$ che trascina e travolgerà eternamente i Paolo e Francesca dell'oggi, del domani e del futuro. Così avviene nel racconto $E l d e$ recho de asilo.

\section{EL DERECHO DE ASILO}

Per quanto riguarda l'«huracán infernal» e gli amori adulteri di Paolo e Francesca ${ }^{52}$ -che sposata nel 1275 a Gianciotto Malatesta, Signore di Rimini, vecchio e deforme, s'innamora di Paolo, fratello del marito e insieme a lui viene ammazzata da questo-, Carpentier prende l'avvio dal notissimo passo dantesco, per compiacersi nella riscrittura di un breve racconto, rivedendo e riproponendo in una nuova cornice, insieme contemporanea e atemporale, la struttura originale.

In El derecho de asilo ${ }^{53}$ Carpentier riporta, sí, l'episodio del canto V dell'Inferno, spoglio, però, dalla presenza di due componenti fondamentali nel testo originale, poiché nella narrazione contemporanea vengono meno sia l'inferno, sia il contrappasso

\footnotetext{
${ }^{47}$ Alejo Carpentier: La consagración de la primavera, Madrid, Siglo XXI de España editores, S.A. 1978 , p. 460

${ }^{48}$ Non è facile, comunque, prendere posizione, se si ascoltano le parole contrastanti dell'autore riguardanti la Commedia: «Eso del amor sublime, inmaterial, es pendejada que inventó Dante, porque tenía fijación con una niña de nueve años llamada Beatriz. Precavido, el poeta prefirió escribir La divina comedia a ser encarcelado por el delito de corrupción de menores». Ibidem, pp. 389-390. «Rabelais, que fue el príncipe de los barrocos franceses, marca la cima de la literatura francesa [...] es evidente que su libro magno del Gargantúa es el único que en la literatura francesa se situa en esa cima de excepciones, de prodigiosos logros donde se encuentran obras como El Quijote, como La divina comedia, o el teatro todo de Shakespeare.» Alejo Carpentier La novela latinoamericana en vísperas de un nuevo siglo y otros ensayos, o. cit., p. 114.

49 [...] Tu non dimandi/ che spiriti son questi che tu vedi? » Inferno IV, vv. 31-32

${ }^{50}$ El arpa y la sombra, o. cit., pp. 213-214. Purgatorio XXX, vv. 73 / 97-100. v. 73 «Guardaci ben! Ben son, ben son Beatrice./[...] lo gel che m'era intorno al cor ristretto,/spirito e acqua fessi, e con angoscia/de la bocca e de li occhi uscì del petto».

51 Ibidem, p. 218 che riprende il verso: «La bufera infernal che mai non resta» Inferno V, 31.

52 Inferno V, vv. 73-138.

53 Alejo Carpentier El derecho de asilo, Barcelona, Ed. Lumen, 1972 . Si cita da: Cuentos completos, Barcelona, Bruguera, 1979 (El derecho de asilo, pp. 181-219).
} 
inflitto a ogni codannato. Non vedremo, quindi, questi moderni amanti esser «menati ${ }^{54}$ dalla violenta bufera, ma li troveremo, tranquilli, seduti nella penombra di una piacevole biblioteca. L'episodio moderno, rivisitato da Carpentier, si svolge fra la caduta di un governo presidenzialista e il consolidamento di uno dei tanti golpes militari che fanno scivolare nel nulla quello stesso governo, e fa espresso riferimento alla storia recente dell' America ispanica. La cronologia della narrazione può quindi essere stabilita in una fascia temporale che si sviluppa fra la seconda decade del ventesimo secolo e momenti, purtroppo, estremamente prossimi. Quanto al contesto geograficospaziale, qualsiasi capitale all'interno di un vasto territorio che si allarga dal «Rio Grande al estrecho de Magallanes» comprese anche determinate isole, è atto a fare da sfondo al racconto.

Prima che l'autore cubano riproponesse, in una nuova riscrittura, la struttura dell' episodio dantesco, la storia di Francesca da Rimini era stata ricordata da drammaturghi quali D'Annunzio ${ }^{55}$ scrittori, fra cui Gustavo Adolfo Bécquer ${ }^{56}$ e Miguel de Unamuno ${ }^{57}$, musicisti quali Ambroise Thomas e Tschaikowsky ${ }^{58}$, oppure poeti come Alfred de Musset $^{59}$, che si rifà a questi versi dell'Inferno per rimpiangere il perduto amore. Ma Carpentier riesce a ricreare un corposo racconto che, nella protetta chiusura di una biblioteca di ambasciata, ci rivela l'idillio nascente fra una ambasciatrice troppo accogliente e un ministro, appena spodestato da un golpe, ospite nell'ambasciata di una nazione amica. La struttura dell'episodio dantesco viene riproposta al lettore umoristicamente, in concomitanza con una nuova fase ${ }^{60}$ della narrativa di Carpentier, che inizia proprio con El derecho de asilo, nella quale all'umore verrà concesso uno spazio molto più largo.

Anche Borges - se tralasciamo considerazioni di ben altro valore riguardanti questo episodio, quale la «paradoja insoluble» per Dante, che «comprende y no perdona» ${ }^{61}$ il peccato di Francesca-, interpreta con umore sfumato la vicenda dei due innamorati. A seguire l'argentino, Dante ${ }^{62}$, cercando di non essere noioso, avrebbe inserito in ogni cerchio dell'Inferno «a un réprobo interesante y no demasiado lejano» e inoltre si sarebbe accorto che «convenía que las confesiones fueran patéticas», in modo da potenziare l'interesse dei lettori.

${ }^{54}$ Per il significato di menare nel canto V, si veda: Paola Marconi, «"Per quello amor che i mena”: Inferno, V, 78 e il Roman de Tristan di Béroul», pp. 77-81.

55 Che compose la tragedia Francesca da Rimini (1901).

56 Rima XXIX «Sobre la falda tenía/el libro abierto/creación de Dante era el libro/era su Infierno», Joaquín Arce «Dante y la lírica de Rubén, Unamuno y Machado» in Literaturas Italiana y Española frente a frente p. 344.

57 «Noi leggevamo un giorno per diletto / di Lancilotto/Fue así, leyendo un libro/ -¡Sempiternos galeotos!,/ fue así; luego subieron de la páginas/ los ojos a los ojos/ y las manos que juntas lo tenían/ se soltaron de pronto.../» Ibidem, p. 340 e anche p.339 e 341.

${ }^{58}$ In seguito alla lettura del passo dantesco compose la Fantasía per gran orchestra, op. 32 chiamata Francesca da Rimini.

59 «Dante, pourqui dis-tu qu'il n'est pire misère qu'un souvenir heurex dans un jour de douleur...» Souvenir, 1841. (E quella a me: «Nessun maggior dolore che ricordarsi del tempo felice ne la miseria; e ciò sa il tuo dottore», Inferno, V, vv.121-123).

${ }^{60}$ Arias, Salvador, «Prólogo» a Recopilación de textos sobre Alejo Carpentier, o. cit., p. 11.

61 Joaquín Arce: Presentación in Jorge Luis Borges, Nueve ensayos dantescos, p. 79.

62 Soprannominato da Borges «El verdugo piadoso». Jorge Luis Borges, Nueve ensayos dantescos, p. 119. 
Non a caso, la biblioteca è largamente fornita di begli esemplari di romanzi di cavalleria: Amadis de Gaula, Palmerín de Hircania e il Caballero Cifar, richiamo non banale a delle tradizioni di letteratura cavalleresca ben note a Dante ${ }^{63}$. E seppure l'ambasciatrice non sembri molto portata per questo tipo di letteratura, il suo compagno cerca di farle capire il piacere che da simili letture si può ricavare, mettendole davanti agli occhi le pagine di Tirante el Blanco, di cui legge un brano scelto a caso. Via via, dalla lettura si sprigiona una dolce voluttuosità che, inconsapevolmente coglie i due lettori. Con dolcezza si giunge pure al momento successivo e sia l'ambasciatrice che il ministro dimenticano il loro ruolo di lettori per diventare soggetti in prima persona del racconto. È qui che il ministro, lasciando scivolare il volume fra le mani, dice: «Y aquel día a fuer de ser pedante, diré «que no leímos más allá”». Col riproporre la traduzione del verso dantesco: Quel giorno piú non vi leggemmo avante ${ }^{64}$, culmina il processo di intertestualità fra uno dei passi più noti dell'Inferno dantesco e il moderno racconto di Alejo Carpentier.

\section{LOS PASOS PERDIDOS}

Per illustrare le scelte dantesche di Carpentier, fin qui abbiamo fatto riferimento a tre opere che risalgono agli anni settanta, ma a chiudere quest'analisi con Los pasos perdidos è d'uopo ritornare indietro di vent'anni, quando, paradossalmente, si conclude, per ciò che riguarda questo breve studio, la via della ricezione dantesca. Siamo del parere che Carpentier ponga fine a questo processo rifacendosi, non al contenuto dell' Inferno bensí al continente, cioè al modello formale, all'involucro imbutiforme, che nel coinvolgere episodi e personaggi, racchiude in se tutta la cantica.

Questo camminare a ritroso non è nuovo per Carpentier che in Viaje a la semilla raccontava già di un uomo: «Don Marcial, Marqués de Capellanías» che, dopo morto, ritorna in vita, percorre verso le origini tutte le tappe precedenti della sua vita, fino al rientro nel calido chiostro materno. E in Los pasos perdidos troviamo ancora un esempio di fuga temporale verso il passato che finisce nella Genesi, ricorsi narrativi fondati sul concetto di reversibilità del tempo. Nell'insieme della sua opera, gli spostamenti avanti e indietro - i corsi e i ricorsi-, sono spesso segnati da riferimenti che, riguardando gli stessi fatti o circostanze, si manifestano riconoscibili in opere cronologicamente distanti, perché in Carpentier c'è pure un'intertestualità interna e tra le sue opere «...se suceden los préstamos textuales, se cruzan los motivos argumentales...» ${ }^{65}$. Si tratta di ripetizioni che nella reiterazione sembrano affievolire o, addirittura sospendere, lo spazio temporale che separa passato, presente e futuro. In parole di Carpentier:

En cierto modo, el Orinoco era para mí una materialización del tiempo en las tres categorías agustinianas: tiempo pasado (tiempo del recuerdo), tiempo presente (tiempo de la in-

${ }^{63}$ Si veda Michelangelo Picone, «Canto V», pp. 85-87.

64 «Quando leggemmo il disïato riso/esser baciato da cotanto amante,/questi, che mai da me non fia diviso,/la bocca mi baciò tutta tremante./Galeotto fu il libro e chi lo scrisse:/ Quel giorno piú non vi leggemmo avante». Inferno, V, vv. 133-138.

65 Padura, o. cit., p. 243. 
tuición), tiempo futuro (tiempo de la espera). Los tres elementos se encontraban para mí presentes en ese río ${ }^{66}$.

In questa «extraña sensación de recurrencia en el tiempo» ${ }^{67}$ pare si produca un processo nel quale non sia impossibile scorgere la fusione tra scene temporalmente e spazialmente determinate. Così le «estampas de infierno» di cui si narra in Los pasos perdidos si sciolgono in quelle di El recurso del método dove il «descenso al infierno» si prospetta come voragine e disordine di suoni, di luce e di forme, nessuno può resistere alla confusione, e tutto è capovolto:

Cuando recuerdo aquel día [...] - confusión de imágenes, descenso al infierno, turbamulta, vocerío sin rumbo, giración de formas, disfraces, metamorfosis, mutaciones, estrépitos sustitución de apariencias, lo de arriba abajo, el buho en mediodía, tinieblas de sol, aparición de harpías, dentelladas de borregos, rugidos del manso, furores del débil; ;.. ${ }^{68}$

E proprio questa nozione di capovolgimento spaziale: «lo de arriba abajo», ci spinge, non senza arditezza, a ipotizzare che nella salita che conduce al Calvario de la Cumbre ${ }^{69}$, illuminata da quindici «focos del alumbrado municipal», si potrebbe, forse, intravedere la rappresentazione formale dell'Inferno, con la sua struttura a imbuto, capovolta. Le allusioni dantesche sembrano percepibili, non soltanto nel definire «estampas de infierno» le scene di vita che si svolgono sotto i fasci di fioca luce sprigionati dai «focos», ma pure nel modo di descrivere il «primer foco» ${ }^{70}$ :

Como los malos siempre arden abajo en toda alegoría de la vida recta y la vida pródiga, el primer foco alumbraba la pulpería de los arrieros, la de los piscos, charandas y aguardientes de berro y mora, lugar de envites y mal ejemplo, con borrachos dormidos sobre los barriles del soportal....

Fermo restando che i poveri ubriachi o le meretrici da due soldi, che popolano il Calvario, non reggono il confronto con i personaggi di Dante, non è meno vero che lo spazio assegnatogli dalla luce di ogni cono, diventato per ognuno degli abitante del Calvario il proprio girone dove scontare, in vita, la condanna della loro esistenza, li accomuna nella tragedia:

...creo de tal manera en la persistencia de ciertas constantes humanas que no veo inconveniente en situar una acción en cualquier momento del pasado puesto que los hombres en todas las épocas han tenido reacciones semejantes ante ciertos acontecimientos. Lo que tú ves como dicotomía, yo lo veo como elemento complementario, como partes de una unidad ${ }^{71}$.

\footnotetext{
${ }^{66}$ Alejo Carpentier: «Un camino de medio siglo», in Razón de ser, Caracas, Ediciones del Rectorado de la Universidad Central de Venezuela. Citato da Alexis Márquez Rodríguez, Lo barroco y lo real maravilloso en la obra de Alejo Carpentier, p. 104

${ }^{67}$ Ibidem, p. 104.

${ }_{68}$ Alejo Carpentier: El recurso del método, Madrid, Siglo XXI Editores. 1974, p. 267. Il corsivo è nostro..

${ }^{69}$ Los pasos perdidos, o. cit., cap. VII, pp. 67-69.

70 Ibidem, p. 67.

71 «Habla Alejo Carpentier», in Recopilación de textos sobre Alejo Carpentier, o. cit., p. 23.
} 
Può darsi che anche il carbonaio che, con sottile premonizione, aveva battezzato il suo carro di lavoro: Los preludios del infierno ${ }^{72}$ avesse subito intuito dove si trovasse, in verità, il luogo dove soffrire le pene del suo contrappasso.

$* \quad * \quad *$

Avviandoci verso una conclusione, preme valutare come e perché sia Carpentier che Bonaviri, più che nelle altre due cantiche della Commedia, si soffermino su certi canti dell'Inferno e vengano proprio a coincidere nel citare il canto XXXIII, dimora dei traditori. Diversi anni fa Bergamin nel saggio citato sulle frontiere infernali della poesia e, quindi, anche su Dante, si esprimeva in questo modo: «Desconfío del lector habitual del Poema Sacro, que le gusta, y prefiere, la lectura del Infierno» ${ }^{73}$. Non direi che sia l'autore cubano che lo scrittore siciliano appartengano alla schiera dei lettori che avrebbero svegliato la diffidenza del saggista spagnolo, direi piuttosto che, attraverso questi esempi di intertestualità dantesca, ci ricordano, in due riletture diverse, la modernità ${ }^{74}$ di Dante e della Commedia. D'altra parte l'Inferno mostra, tuttora, un notevolissimo dinamismo e, non raggiungendo, forse, l'eccellenza e la perfezione del Purgatorio e il Paradiso, riesce a comunicare tutta l'energia rinchiusa ancora nel dolore dei suoi canti, la pietosa carica di angoscia e di infelicità, che ogni uomo può capire, in tempi ed epoche diverse, proprio perché lì si racconta ad infinitum di noi e delle nostre sofferenze.

\section{REFERENCIAS BIBLIOGRÁFICAS}

AAVV (2005): Alejo Carpentier y España. Actas del Seminario Internacional. Santiago de Compostela marzo 2004. Universidade de Santiago de Compostela.

Alonge, Roberto (1997): Luigi Pirandello, Roma, Laterza.

ARCE, Joaquín (1968): «Dante nel Novecento spagnolo», in Il Veltro, XII, 6.

— (1980): «Studi ispano-danteschi: rassegna bibliografico-critica (1965-1977)», in Studi di letteratura italiana in onore di Fausto Montanari, Genova, Il Melangolo.

— (1982): «Dante y la lírica de Rubén, Unamuno y Machado», in Literaturas Italiana y Española frente a frente, Madrid, Espasa Calpe.

ARIAS, Salvador (Ed.) (1977): Recopilación de textos sobre Alejo Carpentier, La Habana, Casa de las Américas.

- (1977): «Prólogo» in Recopilación de textos sobre Alejo Carpentier, pp. 7-13.

Bergamín, José (1980 [1959]): «Dante», en Fronteras infernales de la poesía, Madrid, Taurus, pp. 33-52. In versione italiana: «Dante e la hispanidad», in Maestro Dante, a cura di Vittorio Vettori, Milano, Marzorati Editore, 1962, pp. 108-128.

${ }^{72}$ «Y dejo para lo último el nombre de este carretón de carbonero, cuya revelación me dejó cierta noche absolutamente alucinado: Los preludios del infierno». Alejo Carpentier, La Habana vista por un turista cubano, citato da Padura, o. cit., p. 112.

73 Bergamín, o. cit., p. 44.

${ }^{74}$ La modernità di Dante era stata messa in dubbio da Montale: «Dante non è un poeta moderno.... », in contrasto con il parere di Ungaretti che sosteneva: «A tutt'oggi rimane il più moderno». (Mario Petrucciani «Due paragrafi per Dante e il Novecento», in Silvio Zennaro, Dante nella letteratura italiana del Novecento, p. 164). 
Bonaviri, Giuseppe (1997): Silvinia, Milano, Mondadori.

BorgES, Jorge Luis (1982): Nueve ensayos dantescos. Introducción de Marcos Ricardo Barnatán, Presentación de Joaquín Arce, Madrid, Espasa Calpe.

CARPENTIER, Alejo (1944): Viaje a la semilla, La Habana.

- (1949): El reino de este mundo, México, E.D.I.A.P.S.A.

- (1953): Los pasos perdidos, México, Edición y Distribución Iberoamericana de Publicaciones, Autores Iberoamericanos Contemporáneos.

- (1972): El derecho de asilo, Barcelona, Ed. Lumen.

- (1974): Concierto barroco, Madrid, Siglo XXI Editores.

- (1974): El recurso del método, Madrid, Siglo XXI Editores.

- (1978): La consagración de la primavera, Madrid, Siglo Veintiuno de España editores, S.A.

- (1979): El arpa y la sombra, Madrid, Siglo Veintiuno de España editores, S.A.

- (1979): Bajo el signo de la Cibeles, Recopilador Julio Rodríguez Puértolas, Madrid, Editorial Nuestra Cultura.

- (1981): La novela latinoamericana en vísperas de un nuevo siglo y otros ensayos, Madrid, Siglo XXI Editores.

- (1987), Ese músico que llevo dentro y La música en Cuba [1946], Madrid, Siglo XXI.

Dante Alighieri, La commedia secondo l' antica vulgata, Giorgio Petrocchi (Ed.), Milano, Mondadori, 1966-67, 4 vol.

- La divina commedia, a cura di Natalino Sapegno, Firenze, La Nuova Italia Editrice, $1967^{20}$.

DA VARAZZE, Jacopo (1845): Legenda aurea, vulgo Historia Lombardica dicta, Osnabrück, Otto Zeller Verlag, 1969. Traducción española: Santiago de la Vorágine, La leyenda dorada, Madrid, Alianza Forma, 2 vol.

DURÁN, Manuel (1977): «El cómo y el porqué de una pequeña obra maestra», en Recopilación de textos sobre Alejo Carpentier, pp. 295-320.

FrANK, Janney (1981): Alejo Carpentier and his early works, London, Tamesis Books Limited.

García Carranza, Araceli, (1984): Bibliografía de Alejo Carpentier, La Habana, Letras cubanas.

KuON, Peter (1993): «Lo mio maestro e'l mio autore» Die produktive Rezeption der «Divina Commedia» in der Erzählliteratur der Moderne, Frankfurt am Main, Vittorio Klostermann.

MARCONI, Paola (2002): «"Per quello amor che i mena": Inferno, V, 78 e il Roman de Tristan di Béroul», en L'Alighieri, Ravenna, Angelo Longo Editore, pp. 77-93.

MÁrQUeZ Rodriguez, Alexis (1982): Lo barroco y lo real maravilloso en la obra de Alejo Carpentier, México, Siglo Veintiuno editores.

MARTínez FERnÁndEZ, José Enrique (2001): La intertextualidad literaria, Madrid, Cátedra.

MüLler Bergh, Klaus (1972): Alejo Carpentier: estudio biográfico-crítico, Nueva York, Las Américas.

NAVARro, María Teresa (2002): «Scritture sul mare: Bonaviri e Silvinia», in ...C'era di mezzo il mare: lingua, letteratura e civiltà marina, Firenze, Franco Cesati Editore, pp. 347-360.

PADURA Fuentes, Leonardo (2002): Un camino de medio siglo: Alejo Carpentier y la narrativa de lo real maravilloso, México, Fondo de Cultura Económica.

Picone, Michelangelo (2000): «Canto V», en Lectura Dantis Turicensis. Inferno, a cura di Geroges Güntert e Michelangelo Picone, Firenze, Franco Cesati Editore, pp. 75-89.

Poe, Edgard Allan (1984): Poe. Poetry and Tales, New York, The Library of America, pp. 432-448. Pubblicato per la prima volta nel Graham's Magazine nel maggio del 1841.

Rincón, Carlos (1977): «La poética de lo real-maravilloso americano», en Recopilación de textos sobre Alejo Carpentier, pp. 123-177.

VÁzQUZ Rial, Horacio (1981): Camp de l'arpa. Revista de literatura, 88, Junio. (Monografico su Alejo Carpentier), 
VILLANUEVA, Darío (2005): «La referencia compostelana en la teoría de lo real maravilloso de Alejo Carpentier», en Alejo Carpentier y España, pp. 135-153.

ZAMBORlin, Chiara, (1995): La narrativa di Alejo Carpentier: il mito del mondo migliore, Roma, Bulzoni.

ZENNARo, Silvio (Ed.) (1979): Dante nella letteratura italiana del Novecento Roma, Bonacci Editore. 